\title{
Astrocytes Prevent Ethanol Induced Apoptosis of Nrf2 Depleted Neurons by Maintaining GSH Homeostasis
}

\author{
Madhusudhanan Narasimhan $^{1,2^{*}}$, Marylatha Rathinam ${ }^{1}$, Dhyanesh Patel ${ }^{1}$, \\ George Henderson $^{1,2}$, Lenin Mahimainathan ${ }^{1,2^{*}}$ \\ ${ }^{1}$ Department of Pharmacology and Neuroscience, Texas Tech University Health Sciences Center, Lubbock, USA \\ ${ }^{2}$ South Plains Alcohol and Addiction Research Center, Texas Tech University Health Sciences Center, Lubbock, USA \\ Email: \{1enin.mahimainathan, ${ }^{*}$ madhu.narasimhan\}@ttuhsc.edu \\ Received May 14, 2012; revised June 27, 2012; accepted July 12, 2012
}

\begin{abstract}
Glutathione (GSH), a major cellular antioxidant protects cells against oxidative stress injury. Nuclear factor erythroid 2-related factor 2 (NFE2L2/Nrf2) is a redox sensitive master regulator of battery of antioxidant enzymes including those involved in GSH antioxidant machinery. Earlier we reported that ethanol (ETOH) elicits apoptotic death of primary cortical neurons (PCNs) which in partly due to depletion of intracellular GSH levels. Further a recent report from our laboratory illustrated that ETOH exacerbated the dysregulation of GSH and caspase mediated cell death of cortical neurons that are compromised in Nrf2 machinery (Narasimhan et al., 2011). In various experimental models of neurodegeneration, neuronal antioxidant defenses mainly GSH has been shown to be supported by astrocytes. We therefore sought to determine whether astrocytes can render protection to neurons against ETOH toxicity, particularly when the function of Nrf2 is compromised in neurons. The experimental model consisted of co-culturing primary cortical astrocytes (PCA) with Nrf2 downregulated PCNs that were exposed with $4 \mathrm{mg} / \mathrm{mL}$ ETOH for $24 \mathrm{~h}$. Monochlorobimane (MCB) staining followed by FACS analysis showed that astrocytes blocked ETOH induced GSH decrement in Nrf2-silenced neurons as opposed to exaggerated GSH depletion in Nrf2 downregulated PCNs alone. Similarly, the heightened activation of caspase 3/7 observed in Nrf2-compromised neurons was attenuated when co-cultured with astrocytes as measured by luminescence based caspase Glo assay. Furthermore, annexin-V-FITC staining followed by FACS analysis revealed that Nrf2 depleted neurons showed resistance to ETOH induced neuronal apoptosis when co-cultured with astrocytes. Thus, the current study identifies ETOH induced dysregulation of GSH and associated apoptotic events observed in Nrf2-depleted neurons can be blocked by astrocytes. Further our results suggest that this neuroprotective effect of astrocyte despite dysfunctional Nrf2 system in neurons could be compensated by astrocytic GSH supply.
\end{abstract}

Keywords: Astrocyte-Neuron Co-Culture; Nrf2; Ethanol; Oxidative Stress; GSH

\section{Introduction}

Glutathione (GSH) is the most abundant intracellular non-protein thiol and anti-oxidant in the body with a concentration of approximately $2-3 \mathrm{mM}$ in brain [1]. It is vital for guarding normal healthy metabolism as well as defense against a range of disease and toxicity mechanisms by appropriately controlling cellular redox levels, most notably in the central nervous system (CNS) $[1,2]$. Various studies have demonstrated that maintenance of intracellular GSH pool is important for limiting oxidative-stress induced neuronal injury [3-5]. Several mechanisms underlying GSH synthesis in neurons have been proposed ranging from availability of a rate-limiting substrate, cysteine to $\gamma$-glutamyl cycle that inturn includes a number of enzymes and transporter molecules that all play a vital role in GSH homeostasis $[3,6]$.

\footnotetext{
*Corresponding author.
}

In the last two decades of cellular antioxidant system discoveries, a novel concept of "indirect antioxidants" has been gaining popularity. The factors comprising "Indirect antioxidants" chiefly acts through augmenting cellular antioxidant capacity by binding to antioxidant response elements (ARE) in the promoter of a gene and enhancing its expression. In this class of molecules, transcription factor NF-E2-related factor 2 (NFE2L2/ Nrf2) is demonstrated to be a master regulator activating several antioxidative cytoprotective gene clusters [7]. Activation of Nrf2/ARE pathways has been shown to be neuroprotective when induced by electrophilic drugs [8], growth factors such as bFGF [9], and ethanol [4]. The importance of Nrf2 was further validated using Nrf2 knockout studies in which Nrf2 null animals were found to be more sensitive to various neurotoxins and developed oxidative stress dependent pathological symptoms $[10,11]$. Notably, enzymes involved in GSH homeostasis such as $\gamma$-glutamyl transpeptidase (GGT), glutathione 
synthase, glutathione reductase, $\gamma$-glutamyl cysteine ligase, and multi-drug resistance associated proteins are targets of Nrf2 [7].

Typically, neuronal function in brain is mediated in part by the complex interactions among different cell types including astrocytes. Astrocytes are glial cells that tile up $25 \%$ to $50 \%$ of brain volume outnumbering neurons by over 5:1 [12-14]. These specialized cells lie in close proximity to neurons serving multiple functions ranging from energetic, antioxidant, signaling, plasticity to several others which are required for normal functioning of the nervous system [15-17]. Cortical astrocytes display higher basal and stimulated level of ARE-mediated gene expression than neurons [18,19]. Further cortical astrocytes have been demonstrated to possess stronger and highly regulated Nrf2 dependent GSH homeostasis machinery [20,21]. Initial step in maintenance of neuron GSH homeostasis is efflux of GSH by the astrocyte [22] and the outcome of which is neuroprotection against oxidative damage [23].

Astrocytes can provide direct neurotrophic support to neurons [24]. Reports from numerous laboratories have illustrated that primary neurons co-cultured with astrocytes showed reduced neurotoxicity as compared to neuronal cultures against various neurotoxicants includeing ethanol $[5,23,25]$. However, some studies suggest that neurons become more susceptible to neurotoxicants in the presence of astrocytes [26,27]. Since, astrocytes mediate both positive and negative responses in neuronal cells, neuroscience views normal brain functioning as an outcome of how information processing is accomplished based on neuron-astrocyte interactions. This inturn, is dependent on the diverse physiological and biological responses that is evoked by exogenous-endogenous variables in the individual cellular compartments.

Recent report from our laboratory has shown that ETOH depletes intracellular GSH levels in isolated cortical neuronal cultures without astrocytes [4] (Narasimhan et al., 2011). Further the same study demonstrated that ETOH induced GSH depletion and associated cell death was exaggerated in Nrf2 downregulated primary cortical neurons. In another independent study from our laboratory, we have shown that co-culturing cortical astrocytes with fetal cortical neurons blocked ethanol mediated oxidative stress and normalized GSH homeostasis and subsequent cell death [23]. Thus, in the current study, we have extended our previous observations and attempted to address whether astrocytes can protect neurons even when the latter is compromised in Nrf2 system.

\section{Materials and Methods}

\subsection{Materials}

Minimum Essential Media (MEM), Dulbecco Minimum
Essential Medium (DMEM), Hank's Balanced Salt Solution (HBSS), Fetal Bovine Serum (FBS) were obtained from Invitrogen (Carlsbad, CA). Horse Serum (HS), trypsin, DNase, antibiotics, poly-D-lysine, uridine, monochlorobimane, were purchased from Sigma (St. Louis, MO). Annexin-V FITC apoptosis detection kit was obtained from BD Biosciences (San Jose, CA). Fisher-Costar cell culture inserts for co-culture was from Fisher Scientific (Pittsburgh, PA). Smart Pool siRNA against Nrf2 and non-targeting siRNA pool was purchased from Dharmacon Inc., (Lafayette, CO). Caspase-Glo 3/7 assay kit was obtained from Promega Corporation (Madison, WI). siPORT amine was from Ambion Inc. (Austin, TX).

\subsection{Primary Cortical Neuron (PCN) Cultures}

PCNs were prepared from E16-E17 timed pregnant Sprague Dawley rats as described earlier [28]. Briefly, embryos from amniotic sac were carefully taken out and cerebral cortex from fetus was mechanically dissociated in HBSS. The cells were suspended in MEM containing $10 \%$ FBS and $10 \%$ HS and were grown in a poly-D-lysine coated plates. Cells were maintained in a humidified atmosphere of $95 \%$ air and $5 \% \mathrm{CO}_{2}$. After 1 day in vitro (DIV), the cells were given "inhibitory" feeding with uridine $(10 \mathrm{mg} / \mathrm{mL})$ containing MEM supplemented with $10 \%$ HS to suppress the growth of astrocytes and enrich the cultures for neurons. This is a well-established and documented primary neuronal culture system, which is essentially free of glia. Dual immunostaining with MAP2 (for neurons) and GFAP (for astrocytes) were performed and the isolation procedure reproducibly adopted yielded $\sim 95 \%$ enriched neuronal culture [4,29]. Handling of animals was carried out according to the National Institutes of Health guidelines for the use and care of laboratory animals. The procedures involving isolation of cortical neurons and astrocytes from the embryos were approved by Institutional Animal Care and Use Committee.

\subsection{Small Interfering RNA (siRNA) Transfection}

4 DIV PCNs were transfected with $100 \mathrm{nM}$ of smartpool siRNA against Nrf2 or non-targeting siRNA pool. Briefly, $5 \mu \mathrm{L}$ of siPORT amine and $100 \mathrm{nM}$ of either smartpool siRNA against Nrf2 or non-targeting siRNA pool were diluted in Opti-MEM separately. After complex formation for 20 mins according to manufacturer's instructions (Ambion), the transfection mixture was gently added to PCNs and returned to incubator for $24 \mathrm{~h}$. For the experiments involving ETOH, $24 \mathrm{~h}$ post transfection of siRNA the cells were exposed to ETOH for additional $24 \mathrm{~h}$ and processed for various downstream applications such as FACS analysis for detection of MCB, Annexin V FITC/PI, and the caspase glo assay. 


\subsection{Ethanol Treatment of PCNs}

On 5 DIV, PCNs were treated with ETOH $(4 \mathrm{mg} / \mathrm{mL})$ for $24 \mathrm{~h}$ in an incubator saturated with ETOH to maintain media ETOH (monitored using Analox AM1 alcohol analyzer) [23]. The in vitro experiments involving ETOH in the current study uses a clinically relevant dose, which is at or below to that used in other studies to elicit a range of neurotoxic responses including brain apoptotic responses in various neuron culture, mouse and rat models $[4,23,29,30]$.

\subsection{Primary Cortical Astrocytes (PCA) Cultures}

PCAs were prepared from the cerebral hemispheres of 2-day old rat neonates as described by [31]. Briefly, brain cortices from the new-born pups were aseptically removed, trypsin digested and DNase treated to dissociate the cells. The cells were then filtered through a $0.25 \mu \mathrm{M}$ sieve to remove neurons and the filtered cells containing astrocytes were resuspended in DMEM supplemented with $10 \% \mathrm{FBS}$, antibiotics and seeded in $75 \mathrm{~cm}^{2}$ tissue culture flasks at a density of $6 \times 10^{6}$ cells. The cultures were fed every 3 days and at confluency, the astrocytes were split on day 6 and plated onto $100 \mathrm{~mm}$ petri dish. Approximately $95 \%$ of the cells were identified as astrocytes based on the positivity for glial fibrillary acidic protein (GFAP) staining showing the purity of astrocytic composition of the culture (data not shown).

\subsection{Co-Culture of PCA and Nrf2 siRNA Transfected PCNs}

The transwell insert used in our study is made of polyester membrane of $10 \mu \mathrm{m}$ in thickness with a pore size and diameter of $0.4 \mu \mathrm{m}$ and $24 \mathrm{~mm}$ respectively. These inserts with straight pore structure is suitable and widely employed in transport studies, chemotaxis, co-culture, and microbial pathogenesis studies. For co-culture setting, the cortical astrocytes prepared as above were gently tyrpsinized and replated onto cell-culture inserts at a density of $2.5 \times 10^{5}$ cells/well. The insert plated with astrocytes were further maintained for 7 days to allow it to form a confluent layer across the surface of the insert. Independently, PCNs prepared from E16-E17 as above on 4 DIV were transfected either with smart pool siRNA against Nrf2 or scrambled siRNA pool. On the 5DIV, inserts containing confluent astrocyte cultures were placed in wells containing either Nrf2 siRNA or scramble siRNA transfected neurons and exposed to ETOH (Figure 1(a)). Following $24 \mathrm{~h}$ incubation the cells were processed for various downstream applications such as GSH measurement, caspase $3 / 7$ glo activity assay and apoptosis.

\subsection{GSH Measurement by Flow Cytometry}

Free GSH measurement in live cells was determined by flow cytometry using monochlorobimane, a non-fluorescent reagent which reacts with GSH to form a highly fluorescent derivative [4,32]. At the end of treatment, PCNs were incubated with $10 \mu \mathrm{M}$ of MCB for $30 \mathrm{~min}$ in cell culture incubator, cells scraped, washed, and resuspended in cold PBS. Acquisition and analysis were performed on a FACS flow cytometer with excitation and emission settings of $360 \mathrm{~nm}$ and $460 \mathrm{~nm}$, respectively.

\subsection{Caspase-Glo 3/7 Assay}

Caspases-3/7 activities were estimated using Caspase-glo 3/7. Briefly, at the end of treatment, PCNs were washed with PBS and $300 \mu \mathrm{L}$ of caspase-glo 3/7 reagent was added to each well and the cells were scraped and collected in a microfuge tube in dark. The cell lysate was incubated in dark for $30 \mathrm{~min}$ and the resultant luminescence was read in a Glomax luminometer (Promega). RLU was recorded and results were expressed as fold change in caspase $3 / 7$ activity from control.

\subsection{Annexin-V Staining and FACS Analysis}

Apoptosis was measured using Annexin V binding followed by flow cytometry analysis.

Briefly, both detached and attached cells were harvested and centrifuged at low speed $(1000 \mathrm{~g})$ for $5 \mathrm{~min}$ at the end of the experiment. The cell pellet was washed with cold PBS and resuspended in binding buffer containing annexin V-FITC and propidium iodide (PI). The cells were gently vortexed and incubated in the dark for $15 \mathrm{~min}$. Untreated cells that were either unstained or stained with PI or stained with annexin V-FITC were included along with the experimental samples to correct the background fluorescent signal arising due to the dyes. Data were collected on a flow cytometer and analyzed using Cell Quest (BD) software.

\subsection{Statistical Analysis}

Data are presented as means \pm s.e.m. Statistical differences were determined using one-way ANOVA followed by Student-Newman-Keuls post-hoc analysis and a value of $\mathrm{P}<0.05$ was considered as statistically significant.

\section{Results and Discussion}

\subsection{Co-Culturing Nrf2 Compromised Neurons with Astrocytes Showed Resistance to ETOH Induced GSH Dysregulation}

Generally neurons and astrocytes function as interdependent networks and astrocytes play a critical role in optimizing the function of neurons and protect against diverse insults $[15,17]$. Report from our laboratory demonstrates that astrocytes enable protection of neurons against ETOH by a mechanism that is likely to be based 
(a)
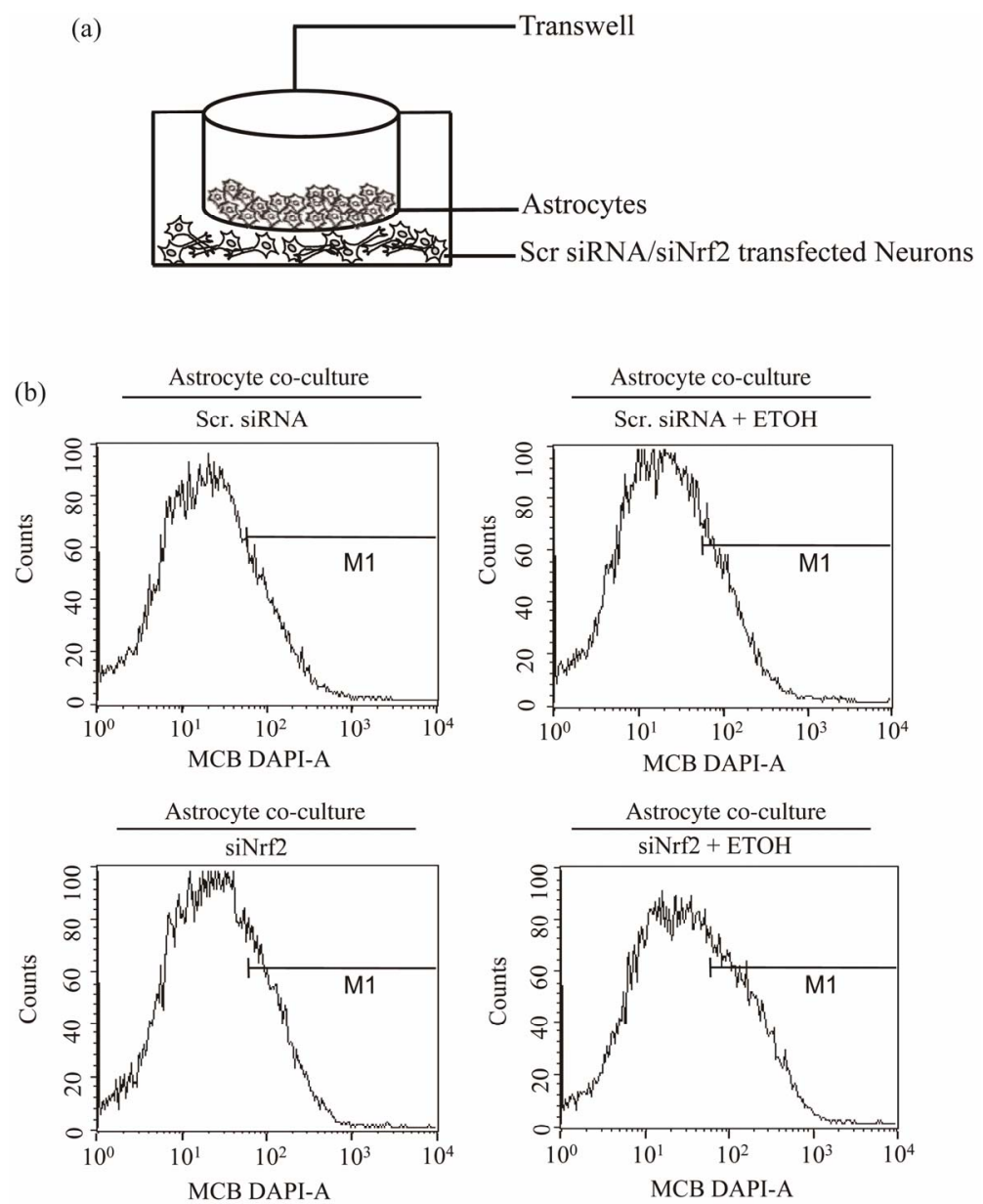

(c)

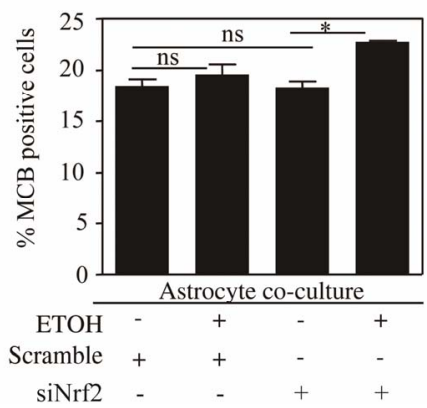

Schematic representation of astrocyte-neuron co-culture set up (a). PCNs transfected either with scramble or Nrf2 siRNA in astrocyte co-culture were exposed to ETOH $(4 \mathrm{mg} / \mathrm{mL})$ for $24 \mathrm{~h}$. At the end of the experiment, neuronal cells were subjected to flow cytometric determination of MCB staining to measure cellular GSH. (a) A representative FACS diagram is shown in panel (b) and panel (c) depict percentage of cells positive for MCB staining. Data are mean \pm s.e.m $(n=3)$ and one-way ANOVA was performed to establish statistical significance. * $: \mathrm{P}<0.05$ and ns represent not significant.

Figure 1. Astrocyte co-culture prevented ETOH induced GSH depletion in Nrf2 silenced primary cortical neurons.

on GSH efflux [23]. Of note, unpublished data from our laboratory suggests that co-culture of neurons with astrocytes deficient in $\mathrm{Nrf2}$, a redox-sensitive regulatory factor controlling GSH homeostasis were unable to offer protection to neurons with intact Nrf2 against ETOH toxicity. Also, perturbation of Nrf2 levels in pure neuronal cultures remarkably sensitizes neurons to $\mathrm{ETOH}-$ induced GSH loss, ROS activation and cell death [4]. Thus we questioned as to whether ETOH exposure to $\mathrm{Nrf} 2$ compromised neurons has any bearing in the GSH antioxidation based health of neurons when co-cultured with astrocytes possessing intact Nrf2. To address this, we utilized Nrf2 targeted primary cortical neurons that are co-cultured with primary cortical astrocytes (PCAs) 
and assessed the effect of ETOH $(4 \mathrm{mg} / \mathrm{mL})$ on intracellular GSH levels in Nrf2 silenced neurons by staining with MCB followed by FACS analysis. Under native state, MCB is non-fluorescent while it only fluoresces upon conjugation to GSH in the cells. Exposure of ETOH to scramble transfected neuron cultures displayed no change in GSH levels when co-cultured with astrocytes (Scr vs Scr + ETOH; Figures 1(b) and (c)). Notably the same concentration of ETOH significantly depleted intracellular GSH levels in PCNs without astrocyte co-culture setting [4]. Importantly, ETOH exposure to Nrf2 silenced neurons showed a significant increase in GSH levels when compared to Nrf2 downregulated neurons (siNrf2 + ETOH vs. siNrf2; Figure 1(c)) as opposed to exaggerated GSH depletion in Nrf2 downregulated primary cortical neurons without astrocyte co-culture [4]. Astroglial cells have been reported to afford extracellular antioxidant protection via GSH efflux as they possess predominant fraction of GSH in brain [33,34]. It has been shown that selective activation of astrocytic Nrf2 protected mice from MPTP-induced Parkinsonism by activating anti- oxidant response pathways [35].

In our previous report, flow cytometric measurement of GSH resulted in a striking heterogeneity of GSH profile viz. high, medium, low GSH containing neurons based on differential MCB staining [4]. However in the current study which involved an astrocyte/neuron coculture setting, we did not observe GSH heterogeneity in neurons. This could be attributed to copious supply of GSH from astrocytes to neurons as GSH efflux from astrocyte is considered to be an initial step in maintenance of neuron GSH homeostasis [23]. It is noteworthy that GSH concentration in neurons was reported to double in $24 \mathrm{~h}$ when co-cultured with astroctyes [36]. Hence it is very much likely that the medium and low GSH population of neurons was supplied with sufficient GSH from astrocytes such that homogeneity in intracellular GSH levels was achieved resulting in uniform population of GSH containing neurons. Thus these results demonstrate that ETOH induced GSH dysregulation in Nrf2-compromised neurons could be controlled when co-cultured with astrocytes via GSH efflux from the latter $[5,23]$. Notably, an ATP-dependent, multidrug-resistant protein 1 (MRP1) in astrocytes have been reported to mediate export of GSH, GSSG and glutathione conjugates [37]. However, the precise mechanism of how GSH effluxes from astrocytes in the current setting remains to be elucidated.

\subsection{Co-Culture of Astrocytes Restrained ETOH Induced Activation of Caspase3/7 Activity in Nrf2 Silenced PCNs}

Progressive cell death is a pathological hallmark of neurodegenerative diseases which in general, is initiated by a cascade of highly organized biochemical, morphological and signaling events involving activation of caspases, a family of cysteine-containing, aspartate specific proteases [38]. Growing body of evidences employ the measure of caspase activity as one of the primary methods to quantify neuronal apoptosis [39]. Among this family of proteases, caspase- 3 and caspase-7 are the effector caspases that executes cell death [40] and thus measurement of effector caspases $3 / 7$ activity is widely gaining popular as index of proapoptotic response $[4,41,42]$. We have demonstrated that ETOH exposure $(4 \mathrm{mg} / \mathrm{mL})$ for $24 \mathrm{~h}$ resulted in a significant increase in caspase $3 / 7$ activity, the effect which was further exacerbated when Nrf2 was downregulated in PCNs [4]. Thus, in the current study we sought to address whether ETOH induced caspase 3/7 activity in Nrf2 downregulated PCNs could be prevented by co-culturing with astrocytes. Treating scramble siRNA-transfected neurons with ETOH when co-cultured with astrocytes did not show any significant activation in caspase 3/7 activity (col. 2 vs. col. 1; Figure 2). Interestingly, ETOH treatment of neurons that are deficient in Nrf2 when co-cultured with astrocytes also failed to show any activation in caspase $3 / 7$ activity (col 4 vs. col. 3; Figure 2). A classical study at the single cell level has demonstrated that execution phase of apoptosis ensues mainly due to depletion of GSH [43]. Induction of oxidative stress has been shown to activate caspase- 3 and 7 resulting in enhancing sensitivity to apoptosis in neurons $[44,45]$. Thus, the observed prevention of ETOH-induced caspase $3 / 7$ activity in Nrf2 depleted neurons could be attributed to abundant supply of GSH from astrocytes that are likely to limit ROS-mediated caspase 3/7 activation. At this juncture, it is important to note that a total downregulation of target gene using knockdown strategy in any primary culture setting is very challenging. In our experimental setting, we could achieve differential knockdown efficiency of Nrf2 ranging between 50\% -

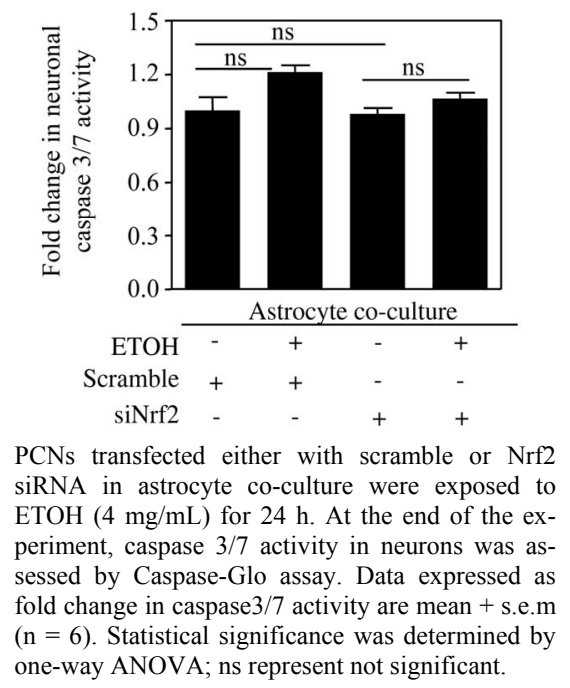

Figure 2. Nrf2 depleted neurons displayed resistance to ETOH-induced caspase 3/7 activation when co-cultured with astrocytes. 
$80 \%$ [4]. Thus, we cannot discount any involvement of residual Nrf2 based antioxidant ac- tivity in neurons that could have also partially contri- buted along with astrocytic supply of GSH to curtail ETOH induced ROS mediated activation of caspase. Nevertheless, this result suggests that normal astrocytes can avert ETOH induced activation of caspase $3 / 7$ asso- ciated apoptotic signaling events in Nrf2-silenced neurons.

\subsection{Astrocytes Co-Culture Protected Nrf2 Silenced PCNs from ETOH Induced Apoptosis}

It is well accepted that astrocytes provide metabolic and trophic support essential for the survival and function of neurons [16,24]. Nrf2 based ARE activation in neighboring astrocytes have been shown to protect neurons against oxidative insult [46]. To determine whether the caspase 3/7 inhibitory effect observed in ETOH exposed
Nrf2-silenced neurons due to astrocyte co-culture was indeed reflecting in controlled apoptosis, we assessed FACS analysis followed by Annexin-V-FITC staining of Nrf2-silenced neurons treated with and without ETOH in astrocyte co-culture set up. In this assay, annexin-V positive cells represent a true state of balance of pro- and anti-apoptotic factors in which greater the positivity, more the shift in balance towards proapoptotic factors typifying early signs of programmed cell death. Neither scramble siRNA nor Nrf2 silenced neurons treated with ETOH when co-cultured with astrocytes displayed any appreciable increase in annexin V-FITC positive (siNrf2 + ETOH vs. siNrf2; Figures 3(a) and (b)). Earlier report from our laboratory has shown that adenovirus mediated overexpression of $\mathrm{Nrf} 2$ in isolated pure neurons prevented ETOH induced apoptosis [4]. However, this study suggests that even when there is a partial loss of $\mathrm{Nrf} 2$ in neurons, astroctyes with intact Nrf2 can curtail (a)
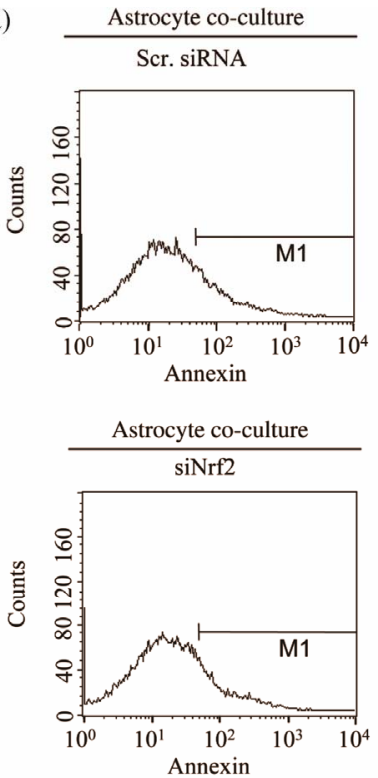
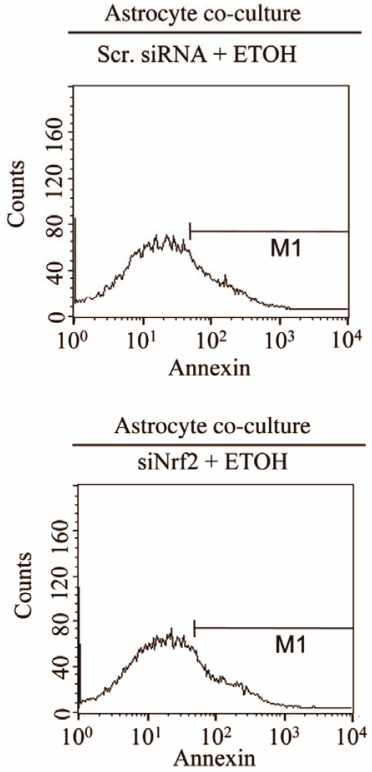

(b)

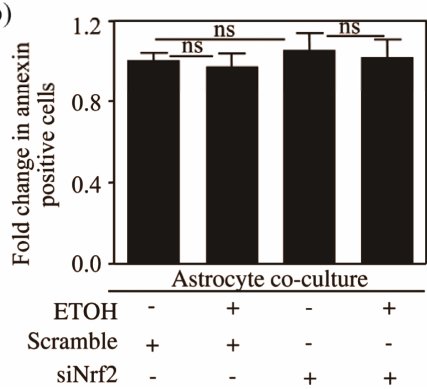

PCNs transfected either with scramble or Nrf2 siRNA in astrocyte co-culture were exposed to ETOH $(4 \mathrm{mg} / \mathrm{mL})$ for $24 \mathrm{~h}$. Neuronal apoptosis was estimated by Annexin-V-FITC staining followed by flow cytometric analysis. (a) Representative FACS diagram is shown in panel (a) and panel (b) depict percentage of cells positive for Annexin-V staining. Data are mean \pm s.e.m $(\mathrm{n}=5)$ and statistical analysis was determined by one-way ANOVA; ns represent not significant.

Figure 3. Astrocytes protected Nrf2 silenced neurons from ETOH induced apoptotic death. 
neuronal apoptosis. In line with our findings, it has been demonstrated that in a mouse model of PD, astrocytic restricted expression of $\mathrm{Nrf} 2$ rendered protection against MPTP mediated neurotoxicity [35]. Furthermore, activation of Nrf2 mediated ATF3 in astrocytes is suggested to afford protection against neurons caused by oxidative stress by modulating redox status and glutathione levels [47]. In the nervous system, ATF-3 is a stress-inducible gene and a transcriptional repressor and considered as a marker of cellular stress [48-50]. ATF3 prevents SCG neuronal cell death and induces neurite elongation via upregulation of Hsp27 after NGF withdrawal [51]. Thus astrocytes could boost antioxidant status in Nrf2 depleted neurons and affords neuronal cytoprotection against ETOH by two probable mechanisms: 1) by functioning as a stress/shock-absorber thus protecting itself against ETOH by activating Nrf2 dependent ATF3 as a counter- response and 2) at the same time generating
GSH in an Nrf2 dependent process and enhancing the availability of GSH to neurons.

In conclusion, the current study identified a neuroprotective role for cortical astrocytes against ETOH even under a condition when neurons are partially depleted of Nrf2 based antioxidant machinery (Figure 4). Another important finding of our study was that this neuroprotective effect of astrocyte despite partially compromised neuronal Nrf2 could be achieved by GSH supply, a central component mediating redox signaling and cell death progression. Studies are underway to identify the appropriate molecular identity of how GSH is transported from astrocytes to neurons.

\section{Acknowledgements}

This work was supported by RO1 AA010114 (to G.I.H). We thank the flow cytometry and optical imaging core facility of UTHSCSA which is supported by NIH-NCI (a)

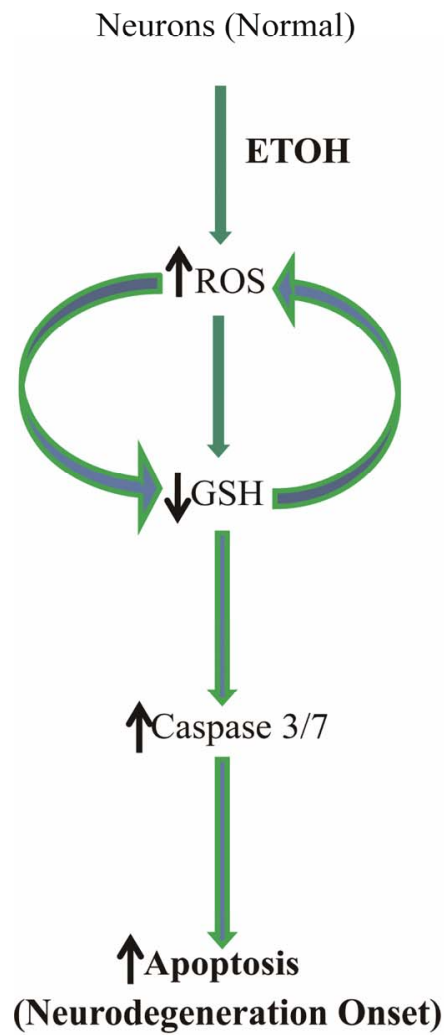

(b)

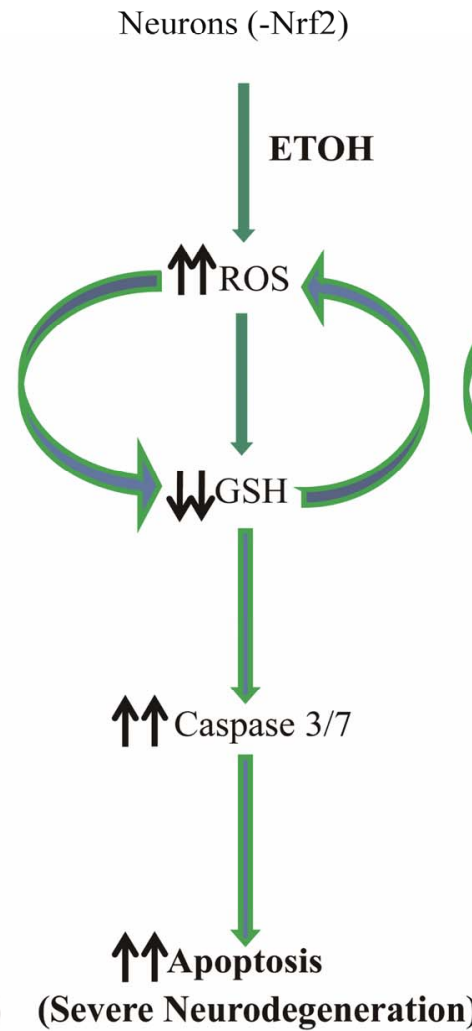

(c)

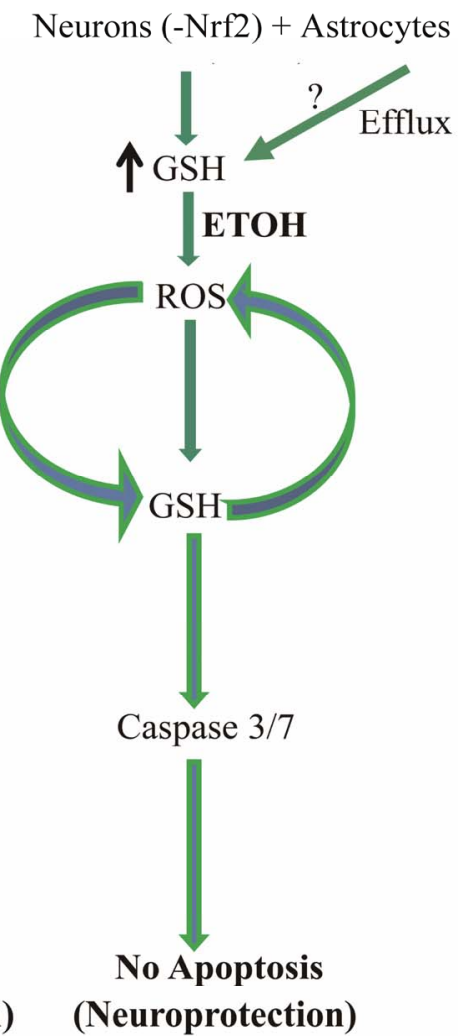

(a) Normal neurons that have low basal Nrf2 levels when exposed to ETOH display a disturbed GSH based redox homeostasis resulting in apoptosis culminating in onset of neurodegeneration [4]; (b) Under conditions when Nrf2 is repressed in neurons, ETOH induced dysregulation in GSH-related apoptotic death is exaggerated resulting in worsening of the neurodegenerative process [4]. Up until second trimester which is when astrocytes colonize the developing CNS, neurons will be highly sensitive to recreational neurotoxicants including, ETOH, a state reflected in Fetal Alcohol Spectrum Disorder (FASD) ((a) and (b)); (c) When Nrf2 compromised neurons are co-cultured with astrocytes, the redox based apoptotic responses to ETOH in neurons is blocked by elevation of neuron GSH levels that is supplied from astrocytes.

Figure 4. Schematic view of astrocytic protection of Nrf2 compromised neurons against ETOH toxicity. 
P30 CA54174 (Cancer Therapy \& Research Center), NIH-NIA P30 AG013319 (Nathan Shock Center) and (NIH-NIA P01AG19316).

\section{REFERENCES}

[1] R. Dringen, "Metabolism and Functions of Glutathione in Brain," Progress in neurobiology, Vol. 62, No. 6, 2000, pp. 649-671. doi:10.1016/S0301-0082(99)00060-X

[2] H. L. Martin and P. Teismann, "Glutathione-A Review on Its Role and Significance in Parkinson's Disease," FASEB Journal: Official Publication of the Federation of American Societies for Experimental Biology, Vol. 23, No. 10, 2009, pp. 3263-3272.

[3] K. Aoyama, M. Watabe and T. Nakaki, "Regulation of Neuronal Glutathione Synthesis," Journal of Pharmacological Sciences, Vol. 108, No. 3, 2008, pp. 227-238. doi:10.1254/jphs.08R01CR

[4] M. Narasimhan, L. Mahimainathan, M. L. Rathinam, A. K. Riar and G. I. Henderson, "Overexpression of Nrf2 Protects Cerebral Cortical Neurons from Ethanol-Induced Apoptotic Death," Molecular Pharmacology, Vol. 80, No. 6, 2011, pp. 988-999. doi:10.1124/mol.111.073262

[5] L. T. Watts, M. L. Rathinam, S. Schenker and G. I. Henderson, "Astrocytes Protect Neurons from Ethanol-Induced Oxidative Stress and Apoptotic Death," Journal of Neuroscience Research, Vol. 80, No. 5, 2005, pp. 655-666. doi:10.1002/jnr.20502

[6] R. Dringen, B. Pfeiffer and B. Hamprecht, "Synthesis of the Antioxidant Glutathione in Neurons: Supply by Astrocytes of CysGly as Precursor for Neuronal Glutathione," The Journal of Neuroscience: The Official Journal of the Society for Neuroscience, Vol. 19, No. 2, 1999, pp. 562-569.

[7] Y. J. Surh, J. K. Kundu and H. K. Na, "Nrf2 as a Master Redox Switch in Turning on the Cellular Signaling Involved in the Induction of Cytoprotective Genes by Some Chemopreventive Phytochemicals," Planta Medica, Vol. 74, No. 13, 2008, pp. 1526-1539. doi:10.1055/s-0028-1088302

[8] T. Satoh, S. I. Okamoto, J. Cui, Y. Watanabe, K. Furuta, M. Suzuki, K. Tohyama and S. A. Lipton, "Activation of the Keap1/Nrf2 Pathway for Neuroprotection by Electrophilic [Correction of Electrophillic] Phase II Inducers," Proceedings of the National Academy of Sciences of the United States of America, Vol. 103, No. 3, 2006, pp. 768773. doi:10.1073/pnas.0505723102

[9] M. R. Vargas, M. Pehar, P. Cassina, L. Martinez-Palma, J. A. Thompson, J. S. Beckman and L. Barbeito, "Fibroblast Growth Factor-1 Induces Heme Oxygenase-1 via Nuclear Factor Erythroid 2-Related Factor 2 (Nrf2) in Spinal Cord Astrocytes: Consequences for Motor Neuron Survival," The Journal of Biological Chemistry, Vol. 280, No. 27, 2005, pp. 25571-25579. doi:10.1074/jbc.M501920200

[10] M. J. Calkins, R. J. Jakel, D. A. Johnson, K. Chan, Y. W. Kan and J. A. Johnson, "Protection from Mitochondrial Complex II Inhibition in Vitro and in Vivo by Nrf2- Mediated Transcription," Proceedings of the National Academy of Sciences of the United States of America, Vol.
102, No. 1, 2005, pp. 244-249.

doi:10.1073/pnas.0408487101

[11] A. Y. Shih, S. Imbeault, V. Barakauskas, H. Erb, L. Jiang, P. Li and T. H. Murphy, "Induction of the Nrf2-Driven Antioxidant Response Confers Neuroprotection during Mitochondrial Stress in Vivo," The Journal of Biological Chemistry, Vol. 280, No. 24, 2005, pp. 22925-22936. doi:10.1074/jbc.M414635200

[12] A. Bignami, "Glial Cells in Central Nervous System," In: P. J. Magistretti, Ed., Discussions in Neuroscience, Elsevier, Amsterdam, 1991, pp. 1-45.

[13] H. K. Kimelberg and M. D. Norenberg, "Astrocytes," Scientific American, Vol. 260, No. 4, 1989, pp. 66-76. doi:10.1038/scientificamerican0489-66

[14] J. O'kusky and M. Colonnier, "A Laminar Analysis of the Number of Neurons, Glia, and Synapses in the Adult Cortex (Area 17) of Adult Macaque Monkeys," The Journal of Comparative Neurology, Vol. 210, No. 3, 1982, pp. 278-290. doi:10.1002/cne.902100307

[15] M. Pekny and M. Nilsson, "Astrocyte Activation and Reactive Gliosis," Glia, Vol. 50, No. 4, 2005, pp. 427-434. doi:10.1002/glia.20207

[16] C. E. Schmidt and J. B. Leach, "Neural Tissue Engineering: Strategies for Repair and Regeneration," Annual Review of Biomedical Engineering, Vol. 5, 2003, pp. 293347.

[17] M. V. Sofroniew and H. V. Vinters, "Astrocytes: Biology and Pathology," Acta Neuropathologica, Vol. 119, No. 1, 2010, pp. 7-35. doi:10.1007/s00401-009-0619-8

[18] D. A. Johnson, G. K. Andrews, W. Xu and J. A. Johnson, "Activation of the Antioxidant Response Element in Primary Cortical Neuronal Cultures Derived from Transgenic Reporter Mice," Journal of Neurochemistry, Vol. 81, No. 6, 2002, pp. 1233-1241. doi:10.1046/j.1471-4159.2002.00913.x

[19] T. H. Murphy, J. Yu, R. Ng, D. A. Johnson, H. Shen, C. R. Honey and J. A. Johnson, "Preferential Expression of Antioxidant Response Element Mediated Gene Expression in Astrocytes," Journal of Neurochemistry, Vol. 76, No. 6, 2001, pp. 1670-1678. doi:10.1046/j.1471-4159.2001.00157.x

[20] J. B. Schulz, J. Lindenau, J. Seyfried and J. Dichgans, "Glutathione, Oxidative Stress and Neurodegeneration," European Journal of Biochemistry/FEBS, Vol. 267, No. 16, 2000, pp. 4904-4911.

[21] A. Y. Shih, D. A. Johnson, G. Wong, A. D. Kraft, L. Jiang, H. Erb, J. A. Johnson and T. H. Murphy, "Coordinate Regulation of Glutathione Biosynthesis and Release by Nrf2-Expressing Glia Potently Protects Neurons from Oxidative Stress," The Journal of Neuroscience: The Official Journal of the Society for Neuroscience, Vol. 23, No. 8, 2003, pp. 3394-3406.

[22] X. F. Wang and M. S. Cynader, "Astrocytes Provide Cysteine to Neurons by Releasing Glutathione," Journal of Neurochemistry, Vol. 74, No. 4, 2000, pp. 1434-1442. doi:10.1046/j.1471-4159.2000.0741434.x

[23] M. L. Rathinam, L. T. Watts, A. A. Stark, L. Mahimainathan, J. Stewart, S. Schenker and G. I. Henderson, 
"Astrocyte Control of Fetal Cortical Neuron Glutathione Homeostasis: Up-Regulation by Ethanol," Journal of Neurochemistry, Vol. 96, No. 5, 2006, pp. 1289-1300. doi:10.1111/j.1471-4159.2006.03674.x

[24] E. D. Martin, A. Araque and W. Buno, "Synaptic Regulation of the Slow $\mathrm{Ca}^{2+}$-Activated $\mathrm{K}^{+}$Current in Hippocampal CA1 Pyramidal Neurons: Implication in Epileptogenesis," Journal of Neurophysiology, Vol. 86, No. 6, 2001, pp. 2878-2886.

[25] K. V. Rao, K. S. Panickar, A. R. Jayakumar and M. D. Norenberg, "Astrocytes Protect Neurons from Ammonia Toxicity," Neurochemical Research, Vol. 30, No. 10, 2005, pp. 1311-1318. doi:10.1007/s11064-005-8803-2

[26] D. R. Brown, "Neurons Depend on Astrocytes in a Coculture System for Protection from Glutamate Toxicity," Molecular and Cellular Neurosciences, Vol. 13, No. 5, 1999, pp. 379-389. doi:10.1006/mcne.1999.0751

[27] L. F. Romao, O. Sousa Vde, V. M. Neto and F. C. Gomes, "Glutamate Activates GFAP Gene Promoter from Cultured Astrocytes through TGF-Beta1 Pathways," Journal of Neurochemistry, Vol. 106, No. 2, 2008, pp. 746-756. doi:10.1111/j.1471-4159.2008.05428.x

[28] V. Ramachandran, L. T. Watts, S. K. Maffi, J. Chen, S. Schenker and G. Henderson, "Ethanol-Induced Oxidative Stress Precedes Mitochondrially Mediated Apoptotic Death of Cultured Fetal Cortical Neurons," Journal of Neuroscience Research, Vol. 74, No. 4, 2003, pp. 577-588. doi:10.1002/jnr.10767

[29] M. Narasimhan, M. Rathinam, A. Riar, D. Patel, S. Mummidi, H. S. Yang, N. H. Colburn, G. I. Henderson and L. Mahimainathan, "Programmed Cell Death 4 (PDCD4): A Novel Player in Ethanol-Mediated Suppression of Protein Translation in Primary Cortical Neurons and Developing Cerebral Cortex," Alcoholism, Clinical and Experimental Research, 2012.

[30] J. W. Olney, T. Tenkova, K. Dikranian, Y. Q. Qin, J. Labruyere and C. Ikonomidou, "Ethanol-Induced Apoptotic Neurodegeneration in the Developing C57BL/6 Mouse Brain," Developmental Brain Research, Vol. 133, No. 2, 2002, pp. 115-126. doi:10.1016/S0165-3806(02)00279-1

[31] K. D. Mccarthy and J. De Vellis, "Preparation of Separate Astroglial and Oligodendroglial Cell Cultures from Rat Cerebral Tissue," The Journal of Cell Biology, Vol. 85, No. 3, 1980, pp. 890-902. doi:10.1083/jcb.85.3.890

[32] S. K. Maffi, M. L. Rathinam, P. P. Cherian, W. Pate, R. Hamby-Mason, S. Schenker and G. I. Henderson, "Glutathione Content as a Potential Mediator of the Vulnerability of Cultured Fetal Cortical Neurons to Ethanol-Induced Apoptosis," Journal of Neuroscience Research, Vol. 86, No. 5, 2008, pp. 1064-1076. doi:10.1002/jnr.21562

[33] R. Dringen, L. Kussmaul, J. M. Gutterer, J. Hirrlinger and B. Hamprecht, "The Glutathione System of Peroxide Detoxification Is Less Efficient in Neurons than in Astroglial Cells," Journal of Neurochemistry, Vol. 72, No. 6, 1999, pp. 2523-2530. doi:10.1046/j.1471-4159.1999.0722523.x

[34] M. L. Schroeter, K. Mertsch, H. Giese, S. Muller, A. Sporbert, B. Hickel and I. E. Blasig, "Astrocytes Enhance
Radical Defence in Capillary Endothelial Cells Constituting the Blood-Brain Barrier," FEBS Letters, Vol. 449, No. 2-3, 1999, pp. 241-244. doi:10.1016/S0014-5793(99)00451-2

[35] P. C. Chen, M. R. Vargas, A. K. Pani, R. J. Smeyne, D. A. Johnson, Y. W. Kan and J. A. Johnson, "Nrf2-Mediated Neuroprotection in the MPTP Mouse Model of Parkinson's Disease: Critical Role for the Astrocyte," Proceedings of the National Academy of Sciences of the United States of America, Vol. 106, No. 8, 2009, pp. 2933-2938. doi:10.1073/pnas.0813361106

[36] S. J. Heales and J. P. Bolanos, "Impairment of Brain Mitochondrial Function by Reactive Nitrogen Species: The Role of Glutathione in Dictating Susceptibility," Neurochemistry International, Vol. 40, No. 6, 2002, pp. 469-474. doi:10.1016/S0197-0186(01)00117-6

[37] J. Hirrlinger, J. B. Schulz and R. Dringen, "Glutathione Release from Cultured Brain Cells: Multidrug Resistance Protein 1 Mediates the Release of GSH from Rat Astroglial Cells," Journal of Neuroscience Research, Vol. 69, No. 3, 2002, pp. 318-326. doi:10.1002/jnr.10308

[38] K. A. Jellinger and C. H. Stadelmann, "The Enigma of Cell Death in Neurodegenerative Disorders," Journal of Neural Transmission, No. 60, 2000, pp. 21-36.

[39] S. Kothakota, T. Azuma, C. Reinhard, A. Klippel, J. Tang, K. Chu, T. J. Megarry, M. W. Kirschner, K. Koths, D. J. Kwiatkowski and L. T. Williams, "Caspase-3-Generated Fragment of Gelsolin: Effector of Morphological Change in Apoptosis," Science, Vol. 278, No. 5336, 1997, pp. 294-298. doi:10.1126/science.278.5336.294

[40] J. G. Walsh, S. P. Cullen, C. Sheridan, A. U. Luthi, C. Gerner and S. J. Martin, "Executioner Caspase-3 and Caspase-7 Are Functionally Distinct Proteases," Proceedings of the National Academy of Sciences of the United States of America, Vol. 105, No. 35, 2008, pp. 1281512819. doi:10.1073/pnas.0707715105

[41] M. C. Kowalczyk, Z. Walaszek, P. Kowalczyk, T. Kinjo, M. Hanausek and T. J. Slaga, "Differential Effects of Several Phytochemicals and Their Derivatives on Murine Keratinocytes in Vitro and in Vivo: Implications for Skin Cancer Prevention," Carcinogenesis, Vol. 30, No. 6, 2009, pp. 1008-1015. doi:10.1093/carcin/bgp069

[42] K. Kumagai, S. Imai, F. Toyoda, N. Okumura, E. Isoya, H. Matsuura and Y. Matsusue, "17 $\beta$-Oestradiol Inhibits Doxorubicin-Induced Apoptosis via Block of the Volume-Sensitive $\mathrm{Cl}^{-}$Current in Rabbit Articular Chondrocytes," British Journal of Pharmacology, Vol. 166, No. 2, 2012, pp. 702-720. doi:10.1111/j.1476-5381.2011.01802.x

[43] R. Franco, M. I. Panayiotidis and J. A. Cidlowski, "Glutathione Depletion Is Necessary for Apoptosis in Lymphoid Cells Independent of Reactive Oxygen Species Formation," The Journal of Biological Chemistry, Vol. 282, No. 42, 2007, pp. 30452-30465. doi:10.1074/jbc.M703091200

[44] H. G. Lee, Y. J. Lee and J. H. Yang, "Perfluorooctane Sulfonate Induces Apoptosis of Cerebellar Granule Cells via a ROS-Dependent Protein Kinase C Signaling Pathway," Neurotoxicology, Vol. 33, No. 3, 2012, pp. 314- 
320. doi:10.1016/j.neuro.2012.01.017

[45] K. Sathishkumar, X. Xi, R. Martin and R. M. Uppu, "Cholesterol Secoaldehyde, an Ozonation Product of Cholesterol, Induces Amyloid Aggregation and Apoptosis in Murine GT1-7 Hypothalamic Neurons," Journal of Alzheimer's Disease: JAD, Vol. 11, No. 3, 2007, pp. 261274.

[46] A. D. Kraft, D. A. Johnson and J. A. Johnson, "Nuclear Factor E2-Related Factor 2-Dependent Antioxidant Response Element Activation by Tert-Butylhydroquinone and Sulforaphane Occurring Preferentially in Astrocytes Conditions Neurons Against Oxidative Insult," The Journal of Neuroscience: The Official Journal of the Society for Neuroscience, Vol. 24, No. 5, 2004, pp. 1101-1112.

[47] K. H. Kim, J. Y. Jeong, Y. J. Surh and K. W. Kim, "Expression of Stress-Response ATF3 Is Mediated by Nrf2 in Astrocytes," Nucleic Acids Research, Vol. 38, No. 1, 2010, pp. 48-59. doi:10.1093/nar/gkp865

[48] B. P. Chen, G. Liang, J. Whelan and T. Hai, "ATF3 and
ATF3 Delta Zip. Transcriptional Repression versus Activation by Alternatively Spliced Isoforms," The Journal of Biological Chemistry, Vol. 269, No. 22, 1994, pp. 1581915826.

[49] T. Hai, C. D. Wolfgang, D. K. Marsee, A. E. Allen and U. Sivaprasad, "ATF3 and Stress Responses," Gene Expression, Vol. 7, No. 4-6, 1999, pp. 321-335.

[50] G. Liang, C. D. Wolfgang, B. P. Chen, T. H. Chen and T. Hai, "ATF3 Gene. Genomic Organization, Promoter, and Regulation," The Journal of Biological Chemistry, Vol. 271, No. 3, 1996, pp. 1695-1701.

[51] S. Nakagomi, Y. Suzuki, K. Namikawa, S. Kiryu-Seo and H. Kiyama, "Expression of the Activating Transcription Factor 3 Prevents c-Jun N-Terminal Kinase-Induced Neuronal Death by Promoting Heat Shock Protein 27 Expression and Akt Activation," The Journal of Neuroscience: The Official Journal of the Society for Neuroscience, Vol. 23, No. 12, 2003, pp. 5187-5196.

\section{Abbreviations}

ETOH, ethanol; PCN, primary cortical neurons; PCA, primary cortical astrocytes; Nrf2/NFE2L2, nuclear factor erythroid 2-related factor 2; GSH, glutathione; MCB, monochlorobimane; siNrf2, small interfering RNA against Nrf2; Scr, Scrambled siRNA. 